\title{
Vertebral augmentation: report of the Standards and Guidelines Committee of the Society of Neurolnterventional Surgery
}

\author{
Ronil V Chandra, ${ }^{1}$ Philip M Meyers, ${ }^{2}$ Joshua A Hirsch, ${ }^{3}$ Todd Abruzzo, ${ }^{4}$ \\ Clifford J Eskey, ${ }^{5}$ M Shazam Hussain, ${ }^{6,7}$ Seon-Kyu Lee, ${ }^{8}$ Sandra Narayanan, ${ }^{9}$ \\ Ketan R Bulsara, ${ }^{10}$ Chirag D Gandhi, ${ }^{11}$ Huy M Do, ${ }^{12}$ Charles J Prestigiacomo, ${ }^{13}$ \\ Felipe C Albuquerque, ${ }^{14}$ Donald Frei, ${ }^{15}$ Michael E Kelly, ${ }^{16}$ William J Mack, ${ }^{17}$ \\ $\mathrm{G}$ Lee Pride, ${ }^{18}$ Mahesh $\mathrm{V}$ Jayaraman, ${ }^{19}$ on behalf of the Society of \\ Neurolnterventional Surgery
}

For numbered affiliations see end of article.

\section{Correspondence to} Dr Mahesh V Jayaraman, Warren Alpert School of Medical at Brown University, Providence, RI 2903, USA; MJayaraman@Lifespan.org

Received 16 October 2013 Accepted 17 October 2013 Published Online First 6 November 2013
To cite: Chandra RV, Meyers PM, Hirsch JA, et al. $J$ Neurolntervent Surg 2014;6:7-15.

\section{INTRODUCTION}

Vertebroplasty and kyphoplasty are minimally invasive image-guided procedures that involve the injection of cement (typically polymethylmethacrylate (PMMA)) into a vertebral body. Kyphoplasty involves inflation of a balloon tamp to create a cavity within the vertebral body into which cement is subsequently injected. The majority of these vertebral augmentation procedures are performed to relieve back pain from osteoporotic or cancerrelated vertebral compression fractures and to reinforce the vertebral body with neoplasm or vascular tumor. The primary goal of vertebroplasty and kyphoplasty is to reduce back pain and to improve patient's functional status, and the secondary goal is stabilization of a vertebra weakened by fracture or neoplasia.

\section{Osteoporotic vertebral fractures}

Osteoporosis is a common disease that causes significant morbidity and incurs a significant healthcare cost to the community. The major osteoporotic fractures involve the hip, vertebra, proximal humerus and distal forearm; the lifetime osteoporotic fracture risk at age 50 is approximately one in two women and one in five men. The lifetime incidence of symptomatic osteoporotic vertebral fractures in women at age 50 is estimated at $10-15 \%{ }^{1}$; once a vertebral fracture occurs, there is a $20 \%$ risk of another vertebral fracture within 12 months. $^{2}$

Most osteoporotic vertebral compression fractures are asymptomatic or result in minimal pain; only a third of vertebral fractures result in medical attention. ${ }^{3}$ Conservative medical therapy is therefore appropriate for the vast majority of vertebral compression fractures since most acute back pain symptoms are mild and subside over a period of $6-$ 8 weeks as the fracture heals. The goals of conservative therapy are pain reduction (with analgesics and/or bed rest), improvement in functional status (with orthotic devices and physical therapy) and prevention of future fractures (with vitamin D, calcium supplementation and antiresorptive agents).

However, conservative treatment for those with severe pain or limitation of function is not benign.
It often involves a period of bed rest that leads to undesirable side effects such as bone mass and muscle strength loss. Markers of bone resorption increase as rapidly as 2 days after bed rest, ${ }^{4}$ with overall bone loss occurring at $1 \%$ per week, 50 times more rapidly than normal age-related bone loss. ${ }^{5}$ After just 10 days of bed rest, healthy older adults lose $15 \%$ of lower extremity strength and $10-15 \%$ of aerobic capacity. ${ }^{6}$ The loss in aerobic capacity is equivalent to almost a decade of age-related decline. ${ }^{6}$ Prolonged bed rest can lead to decubitus ulcers and deep venous thrombosis. Overall, the complications of bed rest combined with narcotic analgesia and associated side effects can result in a vicious cycle of physical deconditioning and subsequent increased risk of additional vertebral insufficiency fractures. These ill effects are particularly pronounced in older osteoporotic patients and can prolong the recovery period and lead to the loss of independence.

In the subset of patients with severe back pain resulting in limitation of function that is refractory to medical therapy, open surgical fixation may be considered but it is often ineffective as the osteoporotic bone may not provide adequate support for surgical hardware. In addition, the elderly population is subject to increased morbidity, mortality and prolonged recovery times. Percutaneous vertebral augmentation, when coupled with appropriate patient selection, is a safe and effective procedure that provides pain reduction and improves functional status. The mechanism of action after PMMA injection is thought to be from mechanical stabilization of mobile fracture fragments and/or thermal or chemical neurolysis; a cadaveric study has also demonstrated new bone formation. ${ }^{7}$

\section{Cancer-related vertebral fractures}

Vertebral fractures in cancer patients may be due to vertebral involvement by tumor, osteopenia caused by androgen deprivation or aromatase inhibitors, or osteonecrosis from radiation therapy. The spine is affected by osteopenic or osteolytic bone disease in approximately $70 \%$ of those with multiple myeloma; new vertebral fractures occur in 15-30\% of patients annually. ${ }^{8}$ In metastatic breast cancer, $17-50 \%$ of patients will sustain a vertebral fracture 
annually and $25-40 \%$ will require radiotherapy for bone pain. ${ }^{8}$ In this cohort of cancer patients, palliative treatments that provide rapid symptom control with improved functional status and quality of life are important.

Open surgical resection and fixation is typically reserved for the minority of patients with focal spinal cord compression, good baseline performance status and reasonable life expectancy. ${ }^{9}$ In a recent systematic review, the response rates (pain relief) measured between 1 week and 1 year showed that palliative radiotherapy reduces back pain in approximately $60 \%$ of patients, with one in four patients reporting complete resolution. ${ }^{10}$ Typically, pain relief begins $7-10$ days after treatment, reaching a maximum between 4 and 8 weeks, so 2 months after radiotherapy has been suggested as the most appropriate time point to measure a meaningful clinical response. ${ }^{11}$ Percutaneous vertebral augmentation is a safe and effective alternative that typically provides rapid pain relief, reduced disability and improved performance status for cancer patients with painful vertebral compression fracture without significant mechanical instability or cord compression. The mechanism of action is thought to be from mechanical stabilization of mobile fracture fragments, thermal or chemical neurolysis or inherent tumoricidal or cytotoxic effects on malignant fractures after PMMA injection. In cases where the diagnosis of tumor is not yet established, another advantage of percutaneous techniques is the ability to perform biopsy at the same time as augmentation.

\section{Purpose of the document}

The purpose of this document is to summarize and classify the evidence for the use of vertebral augmentation in the treatment of patients with osteoporotic or cancer-related vertebral compression fractures. In developing the present recommendations, the Writing Group incorporated already existing guidelines published by the Society of NeuroInterventional Surgery and the American College of Radiology and conducted a systematic review of English language literature published between January 2000 and December 2012 to assess the evidence supporting the use of vertebral augmentation in the treatment of patients with osteoporotic or cancer-related vertebral compression fractures. The Writing Group has applied the rules of evidence and formulation of strength of recommendations used by other American Heart Association (AHA) guideline panels and specifically examined clinical outcome and procedural safety data.

\section{CLINICAL OUTCOME}

Evidence-based medicine involves the integration of the best available evidence with clinical expertise and patient values. The highest level of evidence arises from prospective randomized trials and meta-analysis of these trials. To access clinical outcomes for vertebroplasty and kyphoplasty in painful vertebral compression fractures, we specifically focused on: (1) reduction in back pain; (2) improvement in function; and (3) improvement in quality of life. In particular, we have reviewed both sham controlled studies and conservative management controlled studies which have been published in recent years.

\section{Prospective multicenter randomized sham controlled studies}

The Investigational Vertebroplasty Safety and Efficacy Trial (INVEST) was designed to evaluate the efficacy of PMMA injection in vertebroplasty for patients with painful osteoporotic compression fractures compared with a simulated procedure without PMMA. ${ }^{12}$ As initial recruitment was slow, the power calculations were revised and the target sample size was reduced from 250 to 130 patients. After the first three patients were enrolled, the inclusion criteria were liberalized to back pain intensity of $\geq 3$ (scale $0-10$ ), inadequate pain relief with standard medical therapy and duration of pain of less than 1 year. For fractures of uncertain age, an additional requirement was increased uptake on the bone scan or marrow edema on MRI. Between June 2004 and August 2008, 1813 patients were screened, 1382 patients were not included and 300 patients declined to participate. The remaining 131 patients were randomized to vertebroplasty $(\mathrm{n}=68)$ and sham procedure arms $(n=63)$. The sham procedure involved injection of local anesthetic onto the periosteum of the pedicle as well as verbal and physical cues to simulate vertebroplasty, including pressure on the patient's back and opening of the methacrylate monomer to simulate the odor associated with mixing of PMMA. Overall, mean back pain duration was approximately 18 weeks, with about a third of all patients randomized having pain for $>6$ months.

The primary endpoint was perceived back pain intensity and modified Roland-Morris Disability (RDQ) score at 1 month. There was no significant difference in back pain intensity or modified RDQ score at 1 month $(p=0.19$ and $p=0.49$ at 1 month, respectively). Secondary outcomes of further measures of pain, disability and quality of life also did not differ between the two groups at 1 month. In a post hoc analysis, there was a trend toward a higher rate of clinically meaningful improvement in pain in the vertebroplasty group compared with the control group ( $64 \%$ vs $48 \%, \mathrm{p}=0.06$ ). By 3 months, 27 patients $(43 \%)$ in the control group had crossed over to the other group and thus no longer term comparisons were possible.

A major limitation of the INVEST trial is the inclusion of fractures up to 12 months old, with $36 \%$ of patients in the vertebroplasty arm being treated between 6 and 12 months after the fracture. Marrow edema on MRI or uptake on bone scan was only required for those fractures of indeterminate clinical age; however, the rate of usage of MRI or bone scan was not published. Importantly, the lack of MRI or bone scan correlation could mean that a radiographically occult adjacent level vertebral fracture responsible for the back pain was not treated in the vertebroplasty arm. Alternatively, in the sham procedure arm, infiltration of local anesthetic may have resolved pain that primarily arose from the adjacent structures rather than the fracture itself.

An attempt to address the impact of local anesthetic infiltration in the sham procedure arm was reported in the Local Anesthesia with Bupivacaine and Lidocaine for Vertebral Fracture (LABEL) trial. ${ }^{13}$ Nineteen patients with painful osteoporotic vertebral compression fractures were given an unblinded injection of lidocaine and bupivacaine and compared with 16 patients from the control arm of the INVEST lead site. INVEST control patients had a significantly greater reduction in pain $(\mathrm{p}=0.04)$ and disability $(\mathrm{p}=0.006)$ at day 3 compared with patients in the LABEL trial. This suggests that local anesthetic infiltration did not impact on the outcome and that other factors, including the placebo effect, were responsible for the patient improvement seen in the INVEST control arm. However, there was significant crossover to vertebroplasty in patients in the LABEL trial, with 10 of 19 patients crossing by day 3 and 16 of 19 patients by day 14 . The unblinded nature of the trial may have been biased toward negative patient expectations from local anesthetic injection and positive expectations from vertebroplasty.

The only other multicenter prospective randomized doubleblind clinical trial comparing vertebroplasty with a simulated sham procedure was published contemporaneously with the 
INVEST trial. Buchbinder et al ${ }^{14}$ sought to determine the shortterm efficacy and safety of vertebroplasty for reducing pain and improving physical function in patients with painful osteoporotic vertebral fractures. Inclusion criteria included back pain present for up to 12 months, marrow edema and/or a fracture line on MRI. Between April 2004 and October 2008, 468 patients were screened, 249 were not included and 141 declined to participate. The remaining 78 patients were randomized to vertebroplasty $(n=38)$ and sham procedure $(n=40)$ arms. The sham procedure involved a similar procedure to that in the vertebroplasty group except that the 13-gauge needle was inserted onto the lamina and the central sharp stylet replaced with a blunt stylet. The vertebral body was gently tapped and the PMMA mixed in the room but not injected. Median duration of back pain was approximately 9 weeks in the sample, with 25 patients (one-third of the sample) with symptoms of less than 6 weeks duration.

The primary outcome was back pain score at 3 months. There was no significant difference in back pain scores at 1 week, 1 month, 3 months or at 6 months. Secondary outcome measures of further measures of pain, disability and quality of life also did not differ between the two groups.

Limitations of the trial by Buchbinder et al include the lack of a physical examination component in the methodology and inclusion of fractures up to 12 months old. Although in discussing the primary outcome measure a reduction in back pain score of 1.5 units was regarded as the minimal clinically important difference, the power calculations reported in the publication discuss a sample size of 24 participants in each arm to have $80 \%$ power to show an advantage of vertebroplasty over placebo with respect to pain of at least 2.5 units with a SD of 3 units. In addition, $68 \%$ of patients in the sample were recruited at one of the four recruiting centers with two of the recruiting sites enrolling only five patients, so the outcomes may have been weighted to the treatment effect at this single center.

\section{Prospective multicentre randomized conservative management controlled studies}

The vertebroplasty versus conservative treatment in acute osteoporotic vertebral compression fractures (VERTOS and VERTOS II) trials are the only multicenter prospective randomized clinical trials comparing vertebroplasty with conservative medical management.

The original VERTOS trial was a small prospective multicenter randomized conservative management controlled study to assess the short-term outcome of patients with subacute osteoporotic vertebral compression fractures treated with vertebroplasty compared with conservative management. ${ }^{15}$ Inclusion criteria included debilitating back pain related to the fracture refractory to medical therapy for at least 6 weeks and no longer than 6 months, focal tenderness on physical examination at the affected level and MRI-documented marrow edema, defined as a decreased signal intensity on T1-weighted images and increased signal intensity on short tau inversion recovery (STIR) images. Thirty-four patients were enrolled with 18 patients randomized to vertebroplasty and 16 to conservative management arms. Mean duration of back pain was 11.6 weeks; vertebroplasty was performed within 1 week of enrollment.

The primary endpoint was back pain score (measured by visual analog scale (VAS)) and analgesic use at 1 day and 2 weeks. The differences in the type of analgesic used were quantified by defining analgesic use as an ordinal variable from 0 (no analgesic use), 1 (use of paracetamol), 2 (use of non-steroidal anti-inflammatory drugs) and 3 (use of opiate derivatives). Baseline mean VAS scores were 7.1 and 7.6 in the vertebroplasty and conservative arms respectively; by 1 day the mean VAS scores were 4.7 and 7.1 , respectively, with a significant difference between the two arms of $-2.4(95 \% \mathrm{CI}-3.7$ to -1.0$)$ in favor of vertebroplasty. Analgesic use was also reduced in the vertebroplasty arm (-1.4; $95 \%$ CI -2.1 to -0.8 ). At 2 weeks the difference in the VAS scores was no longer significant.

Secondary outcomes were disability (RMD score) and Quality of Life Questionnaire of the European Foundation for Osteoporosis (QUALEFFO) scores. There were significant improvements in the vertebroplasty arm over conservative management. The mean difference in the RMD scores between the two groups at 2 weeks was -5 points $(95 \%$ CI -8.4 to -1.2 ) while the mean difference in the QUALEFFO scores between the two groups at 2 weeks was -14 points $(95 \% \mathrm{CI}-24.7$ to -3.4$)$.

The limitations of the VERTOS study include the small sample size and lack of blinding. Moreover, as crossover was allowed after 2 weeks, no long-term follow-up was possible as 14 of the 16 patients in the conservative arm requested vertebroplasty.

As there remained no large prospective randomized conservative management controlled study, Klazen et $a l^{16}$ performed the VERTOS II study to assess vertebroplasty with longer term follow-up. Inclusion criteria included severe back pain (VAS score $\geq 5$ ) for 6 weeks or less, focal tenderness at the fracture level and MRI demonstrated bone edema. Of the 934 patients screened, 226 did not meet the inclusion criteria, 232 declined to participate, 229 reported a decrease in pain during screening and thus were not randomized and 45 requested vertebroplasty. The remaining 202 patients were equally randomized into vertebroplasty and conservative medical management arms. All patients were prescribed analgesics that were individually titrated, bisphosphonates, calcium and vitamin D supplements. Vertebroplasty was performed at a mean of 5.6 weeks after symptom onset.

The primary endpoint was pain relief at 1 month and 1 year measured with a VAS score. Vertebroplasty resulted in significantly greater pain relief than conservative treatment. The mean reduction in the VAS score from baseline was 2.6 (95\% CI 1.74 to $3.37, \mathrm{p}<0.0001)$ greater in the vertebroplasty arm at 1 month. This was a durable effect, with a reduction in the mean VAS score at 1 year of $2.0(1.13$ to $2.80, \mathrm{p}<0.0001)$.

The secondary outcome was cost-effectiveness at 1 month and 1 year; medical costs, time without burdensome pain and quality-adjusted survival time were also reported. The cost difference between vertebroplasty and conservative treatment at 1 year was approximately the cost of vertebroplasty for the trial (€2463). An average of 120 (95\% CI 163 to 77) pain-free days were gained in the 12 months after vertebroplasty. Survival analysis revealed that significant pain relief was achieved earlier and in more patients after vertebroplasty (30 days until significant pain relief, 95\% CI 11 to 48$)$ than with conservative treatment (116 days, 95\% CI 86 to 145$)\left(\chi^{2}=55.6, p<0.0001\right)$.

A major limitation to the VERTOS II trial is the lack of blinding, which can overestimate treatment benefit. ${ }^{17}$ Further analysis of the patients in the conservatively treated arm by the VERTOS II investigators revealed that $60 \%$ of patients in the conservatively treated arm had sufficient pain relief (VAS $\leq 3$ ) at 12 months, with the vast majority achieving this within 3 months. ${ }^{18}$ They conclude that vertebroplasty may be appropriate for compression fractures with insufficient pain relief after 3 months.

The Fracture Reduction Evaluation (FREE) trial is the only multicenter prospective randomized clinical trial comparing 
kyphoplasty with conservative medical management. ${ }^{19}$ Inclusion criteria were severe back pain (back pain scale score $\geq 4$ ) for 3 months or less, focal tenderness at the fracture level and MRI demonstrated bone edema, pseudoarthrosis or progressive height loss. Patients with primary or secondary osteoporosis, multiple myeloma or osteolytic metastasis were included. Of the 1279 patients screened, 655 did not meet the inclusion criteria, 209 declined participation and 115 had other reasons for exclusion. The remaining 300 patients were randomized and divided into kyphoplasty $(n=149)$ and conservative medical management $(\mathrm{n}=151)$ arms. All participants received analgesics, bed rest, back braces, physiotherapy, rehabilitation and walking aids according to the practices of participating hospitals. Treatment with calcium, vitamin D and antiresorptive agents was initiated as appropriate. Fractures were a mean of 6 weeks old at randomization, and mean time between randomization and kyphoplasty was 7 days.

The primary endpoint was the difference in change from baseline to 1 month in the global quality of life measure, the Short-Form (SF)-36 physical component summary (PCS) scale. Kyphoplasty resulted in significantly greater improvement in quality of life than did conservative treatment. The improvement in the mean SF-36 PCS score from baseline to 1 month was 5.2 points $(95 \%$ CI 2.9 to 7.4 ) more in the kyphoplasty group than in the conservatively managed arm $(p<0.0001)$.

Secondary outcomes were SF-36 and other quality of life scores, back pain and disability scores at 1, 3, 6 and 12 months. There remained significant improvements in the SF-36 PCS scores in favor of kyphoplasty at 3 and 6 months $(p=0.0008$ and $p=0.0064)$ but not at 12 months $(p=0.208)$. Back pain scores were significantly reduced at 1 week $(p<0.0001)$ and 12 months $(p=0.0034)$. Reductions in the RDQ disability scores in favor of kyphoplasty were significant at 1 month $(\mathrm{p}<0.0001)$ and 12 months $(\mathrm{p}=0.0012)$. Patients undergoing kyphoplasty were less likely to be using narcotic analgesia between 1 and 6 months; these patients also reported 2.9 fewer days of restricted activity per 2 weeks ( 1.3 to $4.6 ; \mathrm{p}=0.0004)$ because of back pain at 1 month, which was no longer significant at 12 months ( 1.6 days, -0.1 to $3.3 ; \mathrm{p}=0.0678$ ).

The FREE trial is limited by inclusion of both osteoporotic and pathological fractures; however, only 4 of the 300 patients randomized had pathological fractures. The trial is also limited by the lack of blinding, which can overestimate treatment benefit. ${ }^{17}$ The FREE investigators have reported 2-year outcome data: although there remained a significant reduction in back pain scores for patients in the kyphoplasty arm compared with conservative therapy at 24 months $(p=0.009)$, there were no significant differences in the SF-36 or RDQ scores at 24 months. $^{20}$

The Cancer Patient Fracture Evaluation (CAFE) trial is the only multicenter prospective randomized clinical trial comparing kyphoplasty with conservative medical management for cancer patients with painful vertebral compression fractures. ${ }^{21}$

Inclusion criteria included a back pain score of $\geq 4$, an RDQ score of at least 10 and vertebral fracture clinically diagnosed in conjunction with either plain x-rays or MRI. Patients with primary bone tumors (eg, osteosarcoma), osteoblastic tumors or a plasmacytoma as the index fracture were excluded. Of the 477 patients screened, 248 were not eligible and 95 refused to participate. The remaining 134 patients were randomized into balloon kyphoplasty $(\mathrm{n}=70)$ and conservative therapy arms $(n=64)$. All patients could receive analgesics, calcium, vitamin D supplements, antiresorptive therapy, bed rest, bracing, physiotherapy, rehabilitation programs, walking aids, radiation treatment and other antitumour therapy at the discretion of treating physicians. Median estimated symptomatic fracture age was 3.5 months (IQR 1.2-6.8); 68\% had edema on MRI.

The primary endpoint was the change in RDQ score at 1 month. Kyphoplasty resulted in a significantly greater reduction in back pain-related disability than did conservative treatment. Mean baseline RDQ scores were 17.6 points in the kyphoplasty group and 18.2 in the control group. By 1 month the mean RDQ score in the kyphoplasty group was 9.1 while the mean RDQ score in the control group was 18.0. The treatment effect for kyphoplasty on RDQ at 1 month was -8.4 points (95\% CI -7.6 to $-9.2 ; \mathrm{p}<0.0001)$. The minimum clinically important difference (MCID) in RDQ ranges between 2 and 3 points. ${ }^{22}$ By 1 month, 51 of 63 patients in the kyphoplasty group improved by at least 2 RDQ points compared with 14 of 50 patients randomly assigned to non-surgical management $(\mathrm{p}<0.0001)$.

Secondary outcomes included back pain scores, Karnofsky performance status (KPS) scores and quality of life (measured by SF-36 PCS) at 1, 3, 6 and 12 months. Patients in the kyphoplasty group had significant reductions in back pain. Both groups had a baseline mean back pain score of 7.3 ; the mean score at 7 days was 3.5 in the kyphoplasty arm compared with 7.0 in the conservative arm $(\mathrm{p}<0.0001)$, and this difference remained of similar magnitude and significant at 1 month $(\mathrm{p}<0.0001)$. The mean baseline KPS score was approximately 56 in both the kyphoplasty and control groups. The MCID estimate for KPS score in cancer patients is about 5 points $^{23} ; 70$ points is a clinically meaningful threshold for self-care. ${ }^{24}$ By 1 month, the mean KPS score in the kyphoplasty group had increased by 15.3 points (95\% CI 13.5 to $17.1 ; \mathrm{p}<0.0001$ ) compared with no significant change in the control group. By 1 month, $75 \%$ of patients in the kyphoplasty group had improved to a KPS score of at least 70 compared with $39 \%$ of the conservative arm. Patients in the kyphoplasty arm also had significant increases in the SF-36 quality of life scores at 1 month $(8.4$ points $(95 \%$ CI 7.7 to $9.1 ; \mathrm{p}<0.0001)$ ) compared with the conservative arm.

A limitation of the CAFE trial is the lack of biopsy of the vertebral fracture. Thus, although patients had a history of cancer, individual fractures may have been caused by metastasis, osteoporosis, radionecrosis or a combination of these etiologies. Nonetheless, $78 \%$ of patients in the kyphoplasty arm had stable or progressive cancer (mainly multiple myeloma or breast cancer) and 34\% had received previous radiation for spinal or bony metastasis. Thus, a high rate of metastatic fractures can be expected. Further limitations include the lack of blinding which can overestimate treatment benefit and the significant crossover from the control group: 34 of 64 patients randomized to the conservative arm crossed over to kyphoplasty, with 21 patients crossing over within 1 week of the 1 -month assessment. Nonetheless, these crossover patient outcomes were separately reported and no patients were allowed to crossover before the 1 -month assessment, so the 1-month outcome measures remain robust.

A systematic review that analyzed mainly non-randomized retrospective observational studies of vertebroplasty in the setting of malignancy published before April 2010 included 987 patients in 30 studies (CAFE trial not included). ${ }^{25}$ Fifteen of these studies ( $n=527$ patients) reported pain scores as an outcome up to 1 month after vertebroplasty. All showed a reduction in pain from baseline levels, with an overall reduction ranging from $20 \%$ to $79 \%$. This was a durable effect in the eight studies ( $n=284$ patients) that reported pain scores 
to 6 months with overall reduction in pain ranging from $47 \%$ to $87 \%$.

\section{Meta-analysis of prospective randomized controlled studies} Anderson et $a l^{26}$ published a recent meta-analysis limited to prospective randomized controlled trials comparing vertebroplasty or kyphoplasty with conservative or sham treatment for osteoporotic fractures. The INVEST, Buchbinder et al, VERTOS, VERTOS II and FREE trials were included, as well as one randomized single-center conservative management controlled trial. Studies that investigated treatment of compression fractures as a result of neoplasm were excluded. As the time points for outcome assessment varied among the studies, outcomes were analyzed as early ( $\leq 12$ weeks) and late ( $\geq 26$ weeks). Overall, the meta-analysis showed greater pain relief, reduced disability and improved quality of life in favor of vertebroplasty in the treatment of symptomatic osteoporotic compression fractures.

The pain VAS standardized mean difference was $0.73(95 \%$ CI 0.35 to 1.10 ) for early (<12 weeks) and 0.58 (95\% CI 0.19 to 0.97$)$ for late time points (6-12 months) favoring vertebroplasty $(\mathrm{p}<0.001)$. No publication bias was identified as the funnel plots for VAS were symmetric.

The RDQ or Oswestry (OSW) was chosen for assessment of spine-specific functional outcome. The standardized mean differences for early and late time points were 1.08 (95\% CI 0.33 to 1.82 ) and 1.16 (95\% CI 0.14 to 1.18 ), respectively. Although there was publication bias by one study identified, the results remained significant even on elimination of this study.

The QUALEFFO and the EQ5-D were chosen as quality of life and health outcome measures. The overall standardized mean difference was 0.39 (95\% CI 0.16 to 0.62 ) at early time points, which decreased to 0.33 (95\% CI 0.16 to 0.51 ) at late time points; these were statistically significant at both time points with no publication bias identified on funnel plots.

\section{Meta-analysis of prospective randomized and non-randomized controlled studies}

Shi et al published a recent meta-analysis limited to prospective randomized and non-randomized controlled trials comparing vertebroplasty with conservative or sham treatment for osteoporotic fractures. ${ }^{27}$ They analyzed the results of nine trials (including the INVEST, Buchbinder et al and VERTOS II trials), with a total of 886 patients. The primary outcome analysis revealed no difference in pain scores between the vertebroplasty group and the sham injection group at 1-29 days and 90 days $(p=0.68$ and 0.29 , respectively). Vertebroplasty resulted in significantly greater pain relief than conservative medical treatment at all time points, including in patients with fractures less than 6 weeks old. The mean difference in the VAS score from baseline was $-2.06(95 \% \mathrm{CI}-3.39$ to -0.74$), \mathrm{p}<0.001)$ in favor of the vertebroplasty arm between 1 and 29 days. This remained significant at 90 and 180 days. For patients with onset of pain of 6 weeks or less, the mean difference in the VAS score from baseline was -2.04 (95\% CI -3.32 to -0.75$), p=0.002)$ in favor of the vertebroplasty arm between 1 and 29 days. There were also significant reductions in disability and improvement in quality of life as measured by the RDQ and QUALEFFO at 30 days in favor of vertebroplasty.

Since publication of the most recent meta-analysis, Blasco et $a l^{28}$ have published a prospective single-center randomized conservative management controlled trial of vertebroplasty in osteoporotic vertebral fractures. Inclusion criteria included back pain score (VAS) of $\geq 4$, less than 12 months duration and the presence of edema on STIR MRI images or increased tracer uptake on bone scan. Two hundred and nineteen patients were screened, 94 were excluded and the remaining 125 patients were randomized to vertebroplasty $(n=64)$ and conservative management $(n=61)$ arms. All patients received analgesics and calcitonin for the first month and began/continued bisphosphonates after the first month. Both groups were offered rescue intrathecal infusion ( $25 \mathrm{mg}$ fentanyl and $1.5 \mathrm{mg}$ bupivacaine) when analgesia was ineffective $(\mathrm{VAS} \geq 7$ ) or if there was intolerance to drug therapy. The mean duration of back pain was 4.7 months; six patients had symptom duration less than 6 weeks.

Primary outcomes were VAS and QUALEFFO scores at 2 weeks, 2 months, 6 months and 12 months. At 2 months there was a significant difference in the mean reduction in VAS scores from baseline in favor of vertebroplasty $(p=0.0172)$. This was not significant at the longer time points. QUALEFFO scores were significant improved compared with baseline at all time points after vertebroplasty but were only significantly improved in the conservative arm at the 6- and 12-month assessments. There was no significant difference in the secondary outcome of analgesic use; of note, rescue intrathecal analgesic therapy was performed in three patients treated with vertebroplasty and in 15 with conservative treatment $(p=0.0015)$. Limitations of the study include the lack of blinding, the crossover rate from conservative to vertebroplasty arms of $11 \%$ and the overall $76 \%$ rate of completion of 12-month follow-up.

\section{PROCEDURAL SAFETY}

The assessment of procedural safety is as important as assessment of efficacy. Both are required to estimate the relative ratio of benefit to harm for treatment of an individual patient. Randomized trials offer the best approach for providing safety data but are limited in the detection of rare harms.

\section{Complications}

The recent meta-analysis by Anderson et $a l^{26}$ was limited to prospective randomized controlled trials comparing vertebroplasty or kyphoplasty with conservative or sham treatment for osteoporotic fractures. The INVEST, Buchbinder et al, VERTOS, VERTOS II and FREE trials were included, as well as one randomized single-center conservative management controlled trial for a total of 612 patients. There were no statistically significant differences in non-procedural adverse events between the conservative and vertebral augmentation arms. Minor procedural complications in the vertebral augmentation arm included asymptomatic cement leaks, soft tissue hematoma, exacerbation of asthma and vasovagal reactions. Major procedural complications in the augmentation arm included one postoperative osteomyelitis following vertebroplasty in a patient who did not receive prophylactic antibiotics and severe radiculopathy secondary to cement leakage that required laminectomy. No death was directly related to either conservative or vertebral augmentation therapy.

The only prospective randomized conservative management controlled clinical trial for patients with malignant painful vertebral compression fractures is the CAFE trial. ${ }^{21}$ Of the 70 patients treated with kyphoplasty, the only procedural complications were one superficial wound infection and one patient with a cement leakage to the adjacent disc who had an adjacent fracture the day after the procedure. ${ }^{21}$ Importantly, patients were excluded if they had vertebral fracture morphology deemed unsuitable as determined by the treating physician. Thus, patients with vertebra plana, comminuted fractures, fractures 
that had posterior wall involvement or those with epidural involvement which would incur higher risk were excluded.

A systematic review that analyzed mainly non-randomized retrospective observational studies of vertebroplasty in the setting of malignancy published before April 2010 included 987 patients in 30 studies. ${ }^{25}$ Five deaths $(0.5 \%)$ that could be attributable to vertebroplasty were reported ( 1 from a cement pulmonary embolus, 2 from chest infections following general anesthesia and 2 from sepsis after emergency spinal decompression. Nineteen patients $(2 \%)$ had severe complications (4 symptomatic pulmonary emboli, 1 hemothorax and 13 patients requiring emergency decompression for hematoma or neuropathy). A meta-analysis using the data was not possible due to the heterogeneity in the patient fracture morphology and types, the use of general anesthesia, the type of intervention performed and the volume of cement injected. Overall, the rate of serious complications ranged between $0 \%$ and $11.5 \%$ and the mortality rate was between $0 \%$ and $7 \%$ across the studies.

\section{Cement leakage}

Extraosseous passage of cement is an important source of complications during vertebral augmentation. The marrow space of the cancellous bone communicates with a network of venous channels. Some of these channels coalesce to form the basivertebral plexus posteriorly in the midline while others traverse small perforations in the cortex about the remainder of the vertebral body margin. These veins communicate with venous plexuses within the epidural space, neural foramina and paraspinal tissue which, in turn, primarily drain into the lumbar and azygous veins. These venous channels are important because they represent possible pathways for extraosseous passage of the PMMA.

Small amounts of cement leakage are common during vertebroplasty for osteoporotic fractures. In VERTOS II, 72\% of treated vertebral bodies demonstrated cement leaks on postprocedural CT, with the majority discal or into segmental veins; none were into the spinal canal. ${ }^{16}$ All patients remained asymptomatic. The overall rate of cement pulmonary emboli as detected by CT in a follow-up cohort was 26\%; all patients were asymptomatic and no patients had reactive pulmonary changes. ${ }^{29}$ The rates of cement leakage were not reported for the INVEST trial. The trial by Buchbinder et al reported minimal cement leakage in 14 of 38 patients $(36 \%),{ }^{14}$ however CT was not used for detection. For kyphoplasty, balloon tamp inflation creates a large low-resistance cavity with cancellous bone compacted around the periphery allowing more viscous cement to be placed at a lower pressure, theoretically resulting in a lower rate of cement leakage. In the FREE trial, cement extravasation occurred in $27 \%$ of treated vertebrae; however, this was assessed with intraoperative fluoroscopy and postoperative x-rays. ${ }^{19}$ Most were endplate or discal leakages and there was one foraminal leakage; none were into the spinal canal and there were no cement embolisms. All patients remained asymptomatic.

A recent retrospective study of CT-guided vertebroplasty for 331 malignant vertebral lesions revealed a local cement leak in $59 \% .^{30}$ Although osteolysis of the posterior wall was evident in $49 \%$ (162 of 331 vertebrae), only 6\% (15 of 331) of leaks were into the spinal canal through the posterior cortex. All leaks were asymptomatic. Pulmonary cement emboli were detected in 1 of $53(2 \%)$ chest $\mathrm{x}$-rays and 10 of $88(11 \%)$ chest CT scans; two patients reported transient chest pain during the procedure but no patients had post-procedural respiratory symptoms. In the CAFE trial, cement leaks were reported in 2 of 70 patients, one of which was discal and associated with adjacent level vertebral body fracture the following day. No patients experienced procedure-related neurological deterioration or pulmonary embolism. $^{21}$

\section{Secondary fractures}

It has been suggested that vertebroplasty and kyphoplasty may increase the risk of new fractures in adjacent vertebrae. The incidence of secondary fractures in the meta-analysis by Anderson et al was not significantly different between the conservative and vertebral augmentation arms. ${ }^{26}$ Of the six trials that reported the incidence of secondary fractures between 2 weeks and 2 years, three studies favored the conservative arm for fewer new vertebral fractures and three favored the vertebroplasty group. Overall, 612 patients were analysed; $18.8 \%$ of patients in the conservative arm developed a secondary fracture between 2 weeks and 2 years compared with $19.4 \%$ in the vertebroplasty arm. The standardized mean effect of $0.064(95 \%$ CI -0.57 to 0.70 ) was not significant.

The meta-analysis by Shi $e a l^{27}$ was limited to prospective randomized and non-randomized controlled trials comparing vertebroplasty with conservative or sham treatment for osteoporotic fractures. They analyzed the results of nine trials, including the INVEST, Buchbinder et al and VERTOS II trials, with a total of 886 patients. There was no difference in the risk of new fractures between the conservative and vertebral augmentation arms $(p=0.82)$. This difference remained insignificant even when trials with sample sizes less than 50 or those not using an intention to treat analysis were excluded. In addition, a funnel plot did not reveal publication bias.

The recent publication by Blasco et $a l^{28}$ of a single-center randomized conservative management controlled trial of vertebroplasty in osteoporotic vertebral fractures was not available at the time of these meta-analyses. They reported the rate of new vertebral fractures on spinal x-rays obtained at 6 and 12 months. New fractures were detected at a higher rate in the vertebroplasty arm than in the conservative arm (OR 2.78; 95\% CI 1.02 to $7.62, \mathrm{p}=0.0462$ ).

\section{All complications}

Overall, major complications occur in less than $1 \%$ of patients treated for osteoporotic compression fractures and in less than $5 \%$ of treated patients with neoplastic involvement. ${ }^{31}$ The rates of clinically evident complications appear to be similar between vertebroplasty and kyphoplasty. Previous practice guidelines for the performance of vertebral augmentation have suggested a threshold all-complication rate of $2 \%$ for treatment of osteoporotic fractures and $10 \%$ for neoplastic fractures. Complication rates above these thresholds should prompt a review of local practice to ascertain the etiology and reduce their incidence.

Although uncommon, the potential complications that should be explained to the patient as part of informed consent include cement leakage; nerve or spinal cord injury resulting in paralysis or bowel/bladder dysfunction or need for emergent decompression; pulmonary embolus (secondary to cement, air or fat emboli); infection (osteomyelitis, epidural abscess); bleeding (vascular injury, paraspinal or soft tissue hematoma; fracture (of rib, pedicle or vertebral body); hypotension or depressed myocardial function (secondary to free methylmethacrylate monomer or fat emboli); pneumothorax (for thoracic levels); and worsened pain or failure to treat. Death from cardiovascular collapse or anaphylaxis to the cement has also been reported. ${ }^{32}$ 


\section{CLINICAL GUIDELINES AND RECOMMENDATIONS}

Based on the critical review of the literature evaluating the evidence supporting the use of vertebral augmentation in the treatment of patients with osteoporotic or cancer-related vertebral compression fractures, the following clinical guidelines for patient care are suggested.

\section{Procedural indications for vertebroplasty and kyphoplasty}

1. Symptomatic osteoporotic or cancer-related vertebral compression fractures refractory to medical therapy.

2. Failure of medical therapy can be defined as

(a) Back pain persisting at a level that prevents ambulation or physical therapy in spite of appropriate analgesic therapy.

(b) Significant side effects of analgesia such as confusion, sedation or severe constipation as a result of analgesic doses required to reduce pain to tolerable levels.

(c) Duration of medical therapy a minimum of 6 weeks.

\section{Absolute contraindications}

1. Active systemic infection, in particular spinal infection.

2. Uncorrectable bleeding diathesis.

3. Insufficient cardiopulmonary health to safely tolerate sedation or general anesthesia.

4. Known allergy to the polymer to be used for the procedure.

\section{Relative contraindications}

1. Significant spinal canal stenosis or compressive myelopathy resulting from the retropulsion of fractured fragment or epidural tumoral extension.

2. Radiculopathy in excess of local vertebral pain.

\section{Technical requirements}

The safe and effective performance of vertebral augmentation requires certain facilities and support personnel. The procedure suite should be large enough to accommodate patient monitoring equipment, image guidance equipment and have cardiopulmonary resuscitation equipment available. The majority of vertebral augmentations can be performed under fluoroscopic guidance and high-quality biplane fluoroscopy is advisable, particularly during cement delivery. Although symptomatic complications of vertebral augmentation are infrequent, there should be rapid availability of CT and/or MRI in case of clinical deterioration.

\section{Pre-procedure evaluation}

Appropriate patient selection is required to identify a patient subset that is likely to benefit from vertebral augmentation and to screen for potential contraindications. The decision to proceed with treatment must be based on the clinical history, physical examination, appropriate laboratory evaluation and spinal imaging.

The results of physical and lower limb neurological examinations should be documented. Point tenderness at the spinous process of the fractured vertebra is the classic physical examination finding. In patients with multiple compression fractures, localization of the acute painful fracture is particularly important to maximize treatment response. In difficult cases, physical examination under fluoroscopic guidance can help localize pain to a specific anatomic level. Baseline lower limb neurological examination is important to facilitate rapid assessment and management of the rare post-procedural alteration of lower limb function.
Imaging of the spine is to be performed in all patients. This is necessary to confirm the clinical diagnosis, aid in the identification and assessment of acuity of the acute painful fracture, identification of potential difficulties and to plan the procedure. $\mathrm{x}$-Rays can serve as the initial imaging evaluation as new compression fractures can be identified when recent prior x-rays are available for comparison. Clefts or intraosseous vacuum phenomena (intravertebral transverse, linear or semilunar radiolucent shadow) may be identified. MRI should be performed on all patients if not contraindicated as this single test provides comprehensive information. MRI distinguishes between benign osteoporotic and pathological fractures, assesses the degree of fracture retropulsion, epidural tumor extension, spinal canal compromise and compression of the spinal cord or nerve roots. Importantly, MRI identifies the unhealed fracture level and also potentially other acute fractures that are not evident on less sensitive modalities such as plain radiography or CT. The single most useful sequence is the STIR or T2-weighted sequence with fat saturation: unhealed fractures show a hyperintense signal consistent with bone marrow. Fracture clefts appear as a linear band of T1 hypointensity and T2 hypointensity or hyperintensity within the vertebral body.

In patients who cannot undergo MRI, a nuclear scintigraphic bone scan is the test of choice to distinguish between healed and unhealed fractures: unhealed fractures take up the injected ${ }^{99 \mathrm{~m}} \mathrm{Tc}-\mathrm{methylene}$ diphosphonate tracer in much higher concentrations. A bone scan is highly predictive of a positive clinical response to vertebral augmentation. ${ }^{33}$ The major disadvantage is the poor spatial resolution that can result in imprecise localization. SPECT imaging can be helpful in this regard, and also is highly predictive of a good clinical response to augmentation. ${ }^{34}$ Bone scan with or without SPECT imaging is limited in not evaluating the spinal canal and its contents.

CT can be used to diagnose unhealed fractures, assess the degree of fracture retropulsion, epidural tumor extension, spinal canal compromise and compression of the spinal cord or nerve roots. Another important utility is the pre-procedural evaluation of the integrity of the posterior vertebral body cortex, particularly in the setting of burst fracture or metastasis. A fracture through the posterior cortex increases the risk of posterior leakage of cement or posterior displacement of bone or tumor during the procedure. In patients with metastatic fractures, CT also helps to define the extent of sclerosis which, in turn, increases the technical challenges associated with the procedure.

If there is a clear disparity between the physical examination findings and imaging or a clear alternative source of back pain, vertebral augmentation should not be performed.

\section{Procedural care}

The local hospital practices and processes regarding confirmation of patient identity, procedure to be performed, procedural site and consent should be followed. It is recommended that the patient's pre-procedural imaging be available within the procedure room to facilitate rapid correlation with imaging performed to ensure that the correct vertebral levels are treated.

In all cases, monitoring of vital signs is essential; pulse oximetry and blood pressure measurement should be performed during delivery of sedation. Drug delivery and monitoring should be performed by certified nursing personnel, nurse anesthetists or anesthesiologists. In the majority of patients, vertebral augmentation can be performed using a combination of local analgesics and moderate sedation. In some cases, general anesthesia is helpful to provide adequate comfort and safety, particularly in patients who are at high risk of airway or 
respiratory complications with prone positioning or those with significant pre-procedural narcotic analgesic requirements. However, having the patient awake is desirable because it allows feedback (eg, increasing pain, neurologic dysfunction) that can alert the operator to potential complications.

\section{Post-procedural care}

All patients should have a period of bed rest and observation after the procedure that can be tailored to the clinical circumstance. Vital signs and lower limb neurological function should be assessed at regular intervals, and supervised ambulation should occur after an appropriate duration of observation. Most patients can be discharged later the same day or can be observed overnight in hospital. In the setting of clinical deterioration suspicious for cement leakage, cross-sectional imaging should be performed and appropriate management rapidly instituted.

Post-procedure follow-up should be performed in the near term. Pain and mobility levels and requirement for analgesia should be assessed and the patient counseled to report any sudden increase in back pain or new back pain as it may indicate a new fracture. Repeat fractures in the treatment cohort are not uncommon, and prevention of future fractures with appropriate medical therapy is particularly important.

\section{RECOMMENDATIONS}

1. Kyphoplasty in selected patients is superior to conservative medical therapy in reducing back pain, disability and improving Karnofsky performance status and quality of life for patients with cancer and disabling back pain from a vertebral fracture (AHA Class IIA, Level of Evidence B).

2. Vertebroplasty and kyphoplasty are reasonable therapeutic options in selected patients with cancer and severe back pain from a vertebral fracture that is refractory to conservative medical therapy (AHA Class IIA, Level of Evidence B).

3. Vertebroplasty and kyphoplasty are reasonable therapeutic options in selected patients with severe back pain from an osteoporotic vertebral fracture that is refractory to conservative medical therapy (AHA Class IIA, Level of Evidence B).

\section{CONCLUSION}

A randomized controlled trial has demonstrated procedural efficacy and safety for kyphoplasty for cancer patients with disabling pain from vertebral fractures, which is consistent with previously published observational data. For osteoporotic fractures there are conflicting data. Although previous observational data were in favor of efficacy for vertebral augmentation, two randomized controlled trials did not show efficacy for vertebroplasty over a sham procedure. Two randomized controlled trials - the VERTOS II and FREE trials-showed efficacy for vertebroplasty and kyphoplasty, respectively, over conservative medical management. All published randomized controlled trials in vertebral augmentation have limitations. A recent meta-analysis of prospective randomized controlled trials comparing vertebroplasty or kyphoplasty with conservative or sham treatment showed evidence in favor of the use of vertebral augmentation with a good safety profile.

Based on the evidence reviewed, clinical guidelines for patient care have been presented. Overall, vertebral augmentation should be limited to selected patients who fail conservative medical management. Further randomized controlled trials of vertebral augmentation in both osteoporotic and malignant disease will improve the strength of evidence available to assess these procedures and help achieve better patient outcomes.

\section{Author affiliations}

${ }^{1}$ Monash Medical Centre, Melbourne, Victoria, Australia

${ }^{2}$ Department of Radiology and Neurological Surgery, Columbia University, New York, New York, USA

${ }^{3}$ Neuro Endovascular Program, Massachusetts General Hospital, Boston,

Massachusetts, USA

${ }^{4}$ Department of Neurosurgery, University of Cincinnati, Cincinnati, Ohio, USA

${ }^{5}$ Department of Radiology, Neurology and Neurosurgery, Dartmouth-Hitchcock

Medical Center, Lebanon, New Hampshire, USA

${ }^{6}$ Cerebrovascular Center, Cleveland Clinic, Cleveland, Ohio, USA

${ }^{7}$ Cleveland Heights, Ohio, USA

${ }^{8}$ Department of Radiology, The University of Chicago, Chicago, Illinois, USA

${ }^{9}$ Departments of Neurosurgery and Neurology, Wayne State University School of Medicine, Detroit, Michigan, USA

${ }^{10}$ Department of Neurosurgery, Yale University School of Medicine, New Haven, Connecticut, USA

${ }^{11}$ New Jersey Medical School, Neurological Institute of New Jersey, Newark, New Jersey, USA

${ }^{12}$ Department of Radiology and Neurosurgery, Stanford University Medical Center, Stanford, California, USA

${ }^{13}$ Department of Neurological Surgery, New Jersey Medical School, University of Medicine and Dentistry of New Jersey, Newark, New Jersey, USA

${ }^{14}$ Division of Neurological Surgery, Barrow Neurological Institute, Phoenix, Arizona, USA

${ }^{15}$ Department of Interventional Neuroradiology, Radiology Imaging Associates,

Englewood, Colorado, USA

${ }^{16}$ Department of Neurosurgery, Royal University Hospital, University of

Saskatchewan, Saskatoon, Saskatchewan, Canada

${ }^{17}$ Department of Neurosurgery, University of Southern California, Los Angeles, California, USA

${ }^{18}$ Department of Neuroradiology, UT Southwestern, Dallas, Texas, USA

${ }^{19}$ Warren Alpert School of Medical at Brown University, Providence, Rhode Island, USA

Contributors All authors contributed to critical review and editing of the manuscript, including review of the results of the pertinent trials as well as the societal recommendations.

\section{Competing interests None.}

Provenance and peer review Not commissioned; internally peer reviewed.

\section{REFERENCES}

1 Johnell O, Kanis J. Epidemiology of osteoporotic fractures. Osteoporosis Int 2005;16 (Suppl 2):S3-7.

2 Lindsay R, Silverman SL, Cooper $C$, et al. Risk of new vertebral fracture in the year following a fracture. JAMA 2001;285:320-3.

3 Cooper C, O'neill T, Silman A. The epidemiology of vertebral fractures. European Vertebral Osteoporosis Study Group. Bone 1993;14(Suppl 1):S89-97.

4 Baecker N, Tomic A, Mika C, et al. Bone resorption is induced on the second day of bed rest: results of a controlled crossover trial. J App/ Physiol 2003;95:977-82.

5 Marie PJ, Kassem M. Extrinsic mechanisms involved in age-related defective bone formation. J Clin Endocrinol Metab 2011;96:600-9.

6 Kortebein P, Symons TB, Ferrando A, et al. Functional impact of 10 days of bed rest in healthy older adults. J Gerontol A Biol Sci Med Sci 2008;63:1076-81.

7 Braunstein V, Sprecher CM, Gisep A, et al. Long-term reaction to bone cement in osteoporotic bone: new bone formation in vertebral bodies after vertebroplasty. J Anat 2008;212:697-701.

8 Mccloskey EV, Guest JF, Kanis JA. The clinical and cost considerations of bisphosphonates in preventing bone complications in patients with metastatic breast cancer or multiple myeloma. Drugs 2001;61:1253-74.

9 Lo SS, Lutz ST, Chang EL, et al. ACR appropriateness criteria spinal bone metastases. J Palliat Med 2012;16:9-19.

10 Chow E, Zeng L, Salvo N, et al. Update on the systematic review of palliative radiotherapy trials for bone metastases. Clin Oncol (R Coll Radiol) 2012;24:112-24.

11 Li KK, Hadi S, Kirou-Mauro A, et al. When should we define the response rates in the treatment of bone metastases by palliative radiotherapy? Clin Oncol ( $R$ Coll Radiol) 2008;20:83-9.

12 Kallmes DF, Comstock BA, Heagerty PJ, et al. A randomized trial of vertebroplasty for osteoporotic spinal fractures. N Engl J Med 2009;361:569-79.

13 Brinjikji W, Comstock BA, Gray L, et al. Local Anesthesia with Bupivacaine and Lidocaine for Vertebral Fracture trial (LABEL): a report of outcomes and comparison with the Investigational Vertebroplasty Efficacy and Safety Trial (INVEST). AJNR Am J Neuroradiol 2010;31:1631-4.

14 Buchbinder $\mathrm{R}$, Osborne $\mathrm{RH}$, Ebeling $\mathrm{PR}$, et al. A randomized trial of vertebroplasty for painful osteoporotic vertebral fractures. N Engl J Med 2009;361:557-68.

15 Voormolen MH, Mali WP, Lohle PN, et al. Percutaneous vertebroplasty compared with optimal pain medication treatment: short-term clinical outcome of patients 
with subacute or chronic painful osteoporotic vertebral compression fractures. The Vertos study. AJNR Am J Neuroradiol 2007;28:555-60.

16 Klazen CA, Lohle PN, De Vries J, et al. Vertebroplasty versus conservative treatment in acute osteoporotic vertebral compression fractures (Vertos II): an open-label randomised trial. Lancet 2010;376:1085-92.

17 Schulz KF, Grimes DA. Blinding in randomised trials: hiding who got what. Lancet 2002:359:696-700.

18 Venmans A, Klazen CA, Lohle PN, et al. Natural history of pain in patients with conservatively treated osteoporotic vertebral compression fractures: results from Vertos II. AJNR Am J Neuroradiol 2012;33:519-21.

19 Wardlaw D, Cummings SR, Van Meirhaeghe J, et al. Efficacy and safety of balloon kyphoplasty compared with non-surgical care for vertebral compression fracture (FREE): a randomised controlled trial. Lancet 2009;373:1016-24.

20 Boonen S, Van Meirhaeghe J, Bastian L, et al. Balloon kyphoplasty for the treatment of acute vertebral compression fractures: 2-year results from a randomized trial. J Bone Miner Res 2011;26:1627-37.

21 Berenson J, Pflugmacher R, Jarzem $\mathrm{P}$, et al. Balloon kyphoplasty versus non-surgical fracture management for treatment of painful vertebral body compression fractures in patients with cancer: a multicentre, randomised controlled trial. Lancet Oncol 2011;12:225-35.

22 Roland M, Fairbank J. The Roland-Morris disability questionnaire and the Oswestry disability questionnaire. Spine 2000;25:3115-24.

23 Ringash J, O'Sullivan B, Bezjak A, et al. Interpreting clinically significant changes in patient-reported outcomes. Cancer 2007;110:196-202.

24 Yates JW, Chalmer B, Mckegney FP. Evaluation of patients with advanced cancer using the Karnofsky performance status. Cancer 1980;45:2220-4.
25 Chew C, Craig L, Edwards R, et al. Safety and efficacy of percutaneous vertebroplasty in malignancy: a systematic review. Clin Radiol 2011;66:63-72.

26 Anderson PA, Froyshteter AB, Tontz WL Jr. Meta-analysis of vertebral augmentation compared to conservative treatment for osteoporotic spinal fractures. J Bone Miner Res 2012:28:372-82.

27 Shi MM, Cai XZ, Lin T, et al. Is there really no benefit of vertebroplasty for osteoporotic vertebral fractures? A meta-analysis. Clin Orthop Relat Res 2012;470:2785-99.

28 Blasco J, Martinez-Ferrer A, Macho J, et al. Effect of vertebroplasty on pain relief, quality of life, and the incidence of new vertebral fractures: a 12-month randomized follow-up, controlled trial. J Bone Miner Res 2012;27:1159-66.

29 Venmans A, Klazen CA, Lohle PN, et al. Percutaneous vertebroplasty and pulmonary cement embolism: results from Vertos II. AJNR Am J Neuroradiol 2010;31:1451-53.

30 Trumm CG, Pahl A, Helmberger TK, et al. Ct fluoroscopy-guided percutaneous vertebroplasty in spinal malignancy: technical results, PMMA leakages, and complications in 202 patients. Skeletal Radiol 2012;41:1391-400.

31 Mcgraw JK, Cardella J, Barr JD, et al. Society of interventional radiology quality improvement guidelines for percutaneous vertebroplasty. J Vasc Interv Radiol 2003; 14:\$311-15.

32 Childers JC Jr. Cardiovascular collapse and death during vertebroplasty. Radiology 2003;228:902; author's reply 902-3.

33 Maynard AS, Jensen ME, Schweickert PA, et al. Value of bone scan imaging in predicting pain relief from percutaneous vertebroplasty in osteoporotic vertebral fractures. AJNR Am J Neuroradiol 2000;21:1807-12.

34 Sola M, Perez R, Cuadras $\mathrm{P}$, et al. Value of bone SPECT-CT to predict chronic pain relief after percutaneous vertebroplasty in vertebral fractures. Spine J 2011;11:1102-7. 